\title{
Coexistence of chaotic and non-chaotic states in the two-dimensional Gauss-Navier-Stokes dynamics
}

\author{
C. Giberti ${ }^{a}$, L. Rondoni ${ }^{b}, *$ C. Vernia ${ }^{c}$ \\ a Dipartimento di Informatica e Comunicazione, Università dell'Insubria, Via Mazzini 5, I-21100 Varese, Italy \\ ${ }^{\mathrm{b}}$ Dipartimento di Matematica and INFM, Politecnico di Torino, Corso Duca degli Abruzzi 24, 1-10129 Torino, Italy \\ ${ }^{c}$ Dipartimento di Matematica, Università di Modena, Via Campi 213/b, 1-41100 Modena, Italy
}

\begin{abstract}
Recently, Gallavotti proposed an Equivalence Conjecture in hydrodynamics, which states that forced-damped fluids can be equally well represented by means of the Navier-Stokes equations (NS) and by means of time reversible modifications of NS called Gauss-Navier-Stokes equations (GNS). This Equivalence Conjecture received numerical support in several recent papers concerning two-dimensional fluid mechanics. The corresponding results rely on the fact that the NS and GNS systems only have one attracting set. Performing similar two-dimensional simulations, we find that there are conditions to be met by the GNS system for this to be the case. In particular, increasing the Reynolds number, while keeping fixed the number of Fourier modes, leads to the coexistence of different attractors. This makes difficult a test of the Equivalence Conjecture, but constitutes a spurious effect due to the insufficient spectral resolution. With sufficiently fine spectral resolution, the steady states are unique and the Equivalence Conjecture can be conveniently established.
\end{abstract}

(C) 2003 Elsevier B.V. All rights reserved.

PACS: 05.70.Ln; 47.27.Ak; 65.50.+m

Keywords: Equivalence of hydrodynamic models; Intermittency

\section{Introduction}

Consider the Navier-Stokes (NS) equation for a two-dimensional incompressible fluid, with periodic boundary conditions. In dimensionless form, this equation can be written as

$\dot{\mathbf{u}}+R^{2}(\mathbf{u} \cdot \partial \mathbf{u})=\Delta \mathbf{u}+\mathbf{f}-\partial p, \quad \operatorname{div}(\mathbf{u})=0$,

where $R$ is the Reynolds number and $\mathbf{f}$ is a time independent forcing term, with vanishing spatial average. As a consequence, the spatial average of the flow $\mathbf{u}$,

* Corresponding author.

E-mail addresses: giberti@uninsubria.it (C. Giberti),

rondoni@polito.it (L. Rondoni), vernia@unimore.it (C. Vernia). is preserved by the dynamics, and can be taken to be zero without loss of generality. Recently, Gallavotti introduced a modification of the NS equation, which takes the form $[1,2]$ :

$\dot{\mathbf{u}}+R^{2}(\mathbf{u} \cdot \partial \mathbf{u})=\alpha \Delta \mathbf{u}+\mathbf{f}-\partial p$,

where $\alpha$ is a time-dependent multiplier, defined in such a way that one global quadratic quantity, such as the enstrophy, is constant in time. In particular, to fix the enstrophy $\mathcal{Q}=\int \boldsymbol{\omega}^{2} \mathrm{~d} x$, where $\boldsymbol{\omega}=\nabla \times \mathbf{u}$ is called vorticity, one must take

$\alpha_{\mathcal{Q}}(\mathbf{u}, \boldsymbol{\omega}, \mathbf{f})=\frac{\int[\boldsymbol{\omega} \cdot \mathbf{f}+\boldsymbol{\omega} \cdot(\boldsymbol{\omega} \cdot \nabla) \mathbf{u}] \mathrm{d} \mathbf{x}}{\int(\nabla \times \omega)^{2} \mathrm{~d} \mathbf{x}}$. 
Eq. (2), with $\alpha$ given by Eq. (3) has been called Gauss-Navier-Stokes (GNS) equation in [2], because $\alpha_{\mathcal{Q}}$ implements Gauss' principle of least constraint [3], in order to make $\mathcal{Q}$ a constant of motion. If Eq. (2) with $\alpha$ given by Eq. (3) correctly describe the behavior of certain fluids, as the results of Refs. [4,5] suggest, those fluids have experimentally observable properties, which the NS equations do not make obvious (cf. Section 2 below). Hence, a thorough examination of Eqs. (2) and (3) becomes necessary.

The numerical solution of the NS and GNS equations with periodic boundary conditions is conveniently performed in terms of the Fourier modes of $\mathbf{u}$, using the truncated expansion:

$$
\begin{aligned}
& \mathbf{u}(\mathbf{x}, t)=\sum_{k \in \mathcal{L}} \gamma_{\mathbf{k}} \exp (\mathrm{ik} \cdot \mathbf{x}) \frac{\mathbf{k}^{\perp}}{|\mathbf{k}|}, \\
& \mathcal{L}=[-L, L] \times[-L, L] \backslash\{\mathbf{0}\} .
\end{aligned}
$$

By $N$ we denote the number of complex modes which corresponds to the chosen $\mathcal{L}$. The numerical solution of Eqs. (1) and (2) is then computed from

$$
\begin{aligned}
\dot{\gamma}_{\mathbf{k}}= & -\beta|\mathbf{k}|^{2} \gamma_{\mathbf{k}}-\mathrm{i} R^{2} \\
& \times \sum_{\substack{\mathbf{k}_{1}+\mathbf{k}_{2}=\mathbf{k}, \mathbf{k}_{1}, \mathbf{k}_{2} \in \mathcal{L}}} \frac{\left(\mathbf{k}_{1}^{\perp} \cdot \mathbf{k}_{2}\right)\left(\left|\mathbf{k}_{2}\right|^{2}-\left|\mathbf{k}_{1}\right|^{2}\right)}{2|\mathbf{k}|\left|\mathbf{k}_{1}\right|\left|\mathbf{k}_{2}\right|} \gamma_{\mathbf{k}_{1}} \gamma_{\mathbf{k}_{2}}+\hat{f}_{\mathbf{k}}
\end{aligned}
$$

$$
\text { for } \mathbf{k} \in \mathcal{L}
$$

obtained substituting (4) in Eqs. (1) and (2), where one takes $\beta=1$ for the NS dynamics, and $\beta=\alpha_{\mathcal{Q}}$, written in terms of Fourier modes, for the GNS dynamics. Because the Fourier modes obey the reality condition $\gamma_{\mathbf{k}}=-\bar{\gamma}_{-\mathbf{k}}$, it suffices to consider the modes $\gamma_{\mathbf{k}}$ with $\mathbf{k} \in \mathcal{L}^{+}=[0, L] \times[-L, L] \backslash\{\mathbf{0}\}$, and to integrate a system of $N$ real ODEs, for the real and imaginary parts of $\gamma_{\mathbf{k}}, x_{\mathbf{k}}$ and $y_{\mathbf{k}}$, respectively, with $\mathbf{k} \in \mathcal{L}^{+}$.

We assume that in $\mathcal{L}^{+}$there is only one nonvanishing $\hat{f}_{\mathbf{k}}$, which is taken equal to 1 and, in our numerical experiments, the non-vanishing mode has either $\mathbf{k}^{*}=(1,-2)$ or $\mathbf{k}^{*}=(2,-1)$. Consider now the global quadratic quantities, defined by

$$
Q_{m}=\sum_{\mathbf{k} \in \mathcal{L}^{+}} \mathbf{k}^{2 m}\left|\gamma_{\mathbf{k}}\right|^{2}, \quad m=0,1,2, \ldots
$$

Then the enstrophy is given by $\mathcal{Q}=2 Q_{1}$, while the energy is defined by $\mathcal{E}=2 Q_{0}$. Recalling the special form of $\mathbf{f}$ which we adopt, and constraining $Q_{1}$ to be a constant under the GNS dynamics, we get

$\beta(\gamma)-\alpha_{\mathcal{Q}}(\gamma)=\frac{\left|\mathbf{k}^{*}\right|^{2} x_{\mathbf{k}^{*}}}{Q_{2}(\gamma)}$, where $x_{\mathbf{k}^{*}}=\mathcal{R} e\left(\gamma_{\mathbf{k}^{*}}\right)$

which must be substituted in Eq. (5). The fourth order Runge-Kutta numerical scheme, which we adopted, does not guarantee that the constraint of constant enstrophy, $\mathcal{Q}(\gamma(t))=\mathcal{Q}_{1}$, be exactly implemented. For this reason, we scale the computed $\gamma$ at every time step, replacing it with $\sqrt{\mathcal{Q}_{1} / \mathcal{Q}(\gamma)} \gamma$, in the surface $\mathcal{Q}(\gamma)=$ $\mathcal{Q}_{1}$. However, this renormalization scheme does not change the convergence order of the original RungeKutta scheme [6]. In our calculations, the time step usually equals $10^{-3}$, except in the most delicate cases where it is reduced to $10^{-4}$. In this paper we denote by $\langle g\rangle_{T}^{\text {NS }}$ the time average of the observable $g$, over $T$ dimensionless time units, under the NS dynamics, and by $\langle g\rangle_{T}^{\mathrm{GNS}}$ the corresponding time average under the GNS dynamics. The limits of these averages for growing $T$ are denoted by $\langle g\rangle^{\mathrm{NS}}$ and $\langle g\rangle^{\mathrm{GNS}}$, respectively.

\section{Equivalence}

The first set of numerical experiments which we discuss concerns the role of the initial condition $\mathbf{u}^{\mathrm{GNS}}(0)$ for the GNS evolution, when $\mathbf{u}^{\mathrm{GNS}}(0)$ is the last field $\mathbf{u}^{\mathrm{NS}}(T)$ produced by a long NS evolution which has reached a non-equilibrium steady state. In particular, we consider the NS evolution over a number of different time intervals $\left[0, T_{j}\right], j=1, \ldots, n_{i c}$, starting from the same initial field $\mathbf{u}^{\mathrm{NS}}(0)$, and with $T_{j}>T$ for all $j$, where $T$ is the time needed to reach a steady state. We then simulate $n_{i c}$ GNS evolutions, taking $\mathbf{u}_{j}^{\mathrm{GNS}}(0)=$ $\mathbf{u}^{\mathrm{NS}}\left(T_{j}\right)$ as initial condition for the $j$ th GNS run. This way, each different GNS evolution takes place on a different surface $\mathcal{Q}(\gamma)=\mathcal{Q}_{1}\left(\mathbf{u}^{\mathrm{NS}}\left(T_{j}\right)\right)$ and yields a different value for the time average of the term $\alpha_{\mathcal{Q}}$.

In our framework, the Equivalence Conjecture proposed by Gallavotti in [2], and later revised and tested in Refs. [4,5], can be stated as follows. 
Equivalence Conjecture. The stationary phase space probability distributions of the NS equations and of the GNS equations are equivalent in the limit of large Reynolds number, provided the fixed enstrophy $\mathcal{Q}$ of the GNS evolution coincides with the average enstrophy $\langle\mathcal{Q}\rangle^{\mathrm{NS}}$ of the NS evolution at the same $R$.

In analogy with the theory of equivalence of equilibrium ensembles, the object of the Equivalence Conjecture are the "local observables", i.e. the quantities which depend only on a finite number of Fourier modes of the velocity field $\mathbf{u}$. In other words, if the Equivalence Conjecture is verified and $\varphi$ is a local observable, the ratio $\langle\varphi\rangle^{\mathrm{NS}} /\langle\varphi\rangle^{\mathrm{GNS}}$ should converge to 1 as $R$ tends to infinity. Refs. [4,5] showed that the equivalence may concern also global quantities. In particular, one expects $\left\langle\alpha_{\mathcal{Q}}\right\rangle^{\mathrm{GNS}}=1$. i.e. that the average "viscosity" of the GNS evolution equals the viscosity of the NS equation. This fact, first observed in most of the cases treated in [4], was made precise in [5] for the NS and GNS dynamics with only one steady state, and it is studied in a different setting in this paper. In particular, we investigate the dependence of $\left\langle\alpha_{\mathcal{Q}}\right\rangle_{T}^{\mathrm{GNS}}$ on the difference $\mathcal{Q}\left(\mathbf{u}^{\mathrm{NS}}\left(T_{j}\right)\right)-\langle\mathcal{Q}\rangle_{T}^{\mathrm{NS}}$ in order to check whether it is the case or not that $\left\langle\alpha_{\mathcal{Q}}\right\rangle_{T}^{\mathrm{GNS}} \simeq 1$, whenever $\mathcal{Q}\left(\mathbf{u}^{\mathrm{NS}}\left(T_{j}\right)\right) /\langle\mathcal{Q}\rangle_{T}^{\mathrm{NS}} \simeq 1$.

The interest of the Equivalence Conjecture lies in the following facts. If the NS and GNS description of the same fluid are equivalent, one may use one equation or the other, depending on the particular features of the fluid one wishes to describe. For instance, if the GNS equation is valid, the dissipated power in the fluid fluctuates, and its fluctuations could be described by the Gallavotti-Cohen fluctuation theorem [7]. This property is not evidenced by the NS equation, which predicts that the dissipated power is constant in time $[2,4,5] .^{1}$ At the same time, a local fluctuation theorem can be derived for the same systems for which the Gallavotti-Cohen theorem holds (see, e.g. Ref. [8]). Therefore, one may think that this local theorem holds for the GNS dynamics and, by virtue of the Equiv-

\footnotetext{
${ }^{1}$ Note that there is no conflict between the predictions of the NS and GNS equations, in this instance, because the fluctuations which are the object of the Gallavotti-Cohen theorem should not be observed, normally, in macroscopic systems.
}

alence Conjecture, that it must hold also for the NS dynamics. As a matter of fact, the results of Ref. [5] indicate that this is the case. The Equivalence Conjecture, then, appears as a useful theoretical tool for the extension of the theory of equivalence of ensembles to non-equilibrium statistical mechanics, for a deeper understanding of the properties of the NS dynamics, and also for suggesting new experiments, like those reported in Refs. [9,10].

The idea behind the Equivalence Conjecture is simple. If the fluctuations of $\alpha_{\mathcal{Q}}$ occur on time scales which are short compared with the macroscopic observation time scales, and if $\left\langle\alpha_{\mathcal{Q}}\right\rangle^{\mathrm{GNS}}=1$, the macroscopic observables should be equally well computed from the NS and the GNS dynamics. However, $\left\langle\alpha_{\mathcal{Q}}\right\rangle^{\mathrm{GNS}}$ cannot be adjusted at will, because it is automatically produced by the GNS evolution, and the viscosity of the NS equation cannot be adjusted either, as it is fixed to be 1. The Equivalence Conjecture allows us to circumvent this difficulty, suggesting that the equality of the average viscosities is obtained if the average enstrophies are equal. When the value of $\langle\mathcal{Q}\rangle^{\mathrm{NS}}$ is known, the equality $\langle\mathcal{Q}\rangle^{\mathrm{NS}}=\mathcal{Q}_{\mathrm{GNS}}$ can be easily imposed in various ways, e.g. following the procedure of $[4,5]$ or simply taking at random an initial velocity field for the GNS evolution, and then rescaling this field so that its initial enstrophy equals $\langle\mathcal{Q}\rangle^{\mathrm{NS}}$. Once the equality of the average enstrophies is obtained, the large $R$ limit is required to ensure that the fluctuations of $\alpha_{\mathcal{Q}}$ do really occur on sufficiently short time scales, and the results of $[4,5]$ suggest that this is the case if the initial fields of the GNS evolutions are properly constructed. However, the question of the influence of the GNS initial fields on the validity of the Equivalence Conjecture has not been investigated in [4,5]. This question becomes important if the phase spaces of the NS or GNS equations contain more than one steady state. In that case, tuning the parameters of the NS and GNS evolutions as required by the Equivalence Conjecture may not be enough to obtain the desired equivalence, because the initial conditions of the NS and of the GNS evolutions may fall into attractors with different statistical properties. In this paper, we will observe that this, indeed, does happen in certain cases 
and we will characterize the cases for which it does not.

\section{Results}

Figs. 1 and 2 portray the typical probability distribution $\pi_{R}$ of the quantity defined by

$q=\mathcal{Q}\left(\mathbf{u}^{\mathrm{NS}}\right) /\langle\mathcal{Q}\rangle_{T}^{\mathrm{NS}}$

for $N=24$ and $R^{2}=200,2000$. These distributions are constructed using the fields $\mathbf{u}^{\mathrm{NS}}\left(T_{j}\right), j=$ $1, \ldots, n=5 \times 10^{4}$, produced by the NS evolution at the time instants $T_{1}, T_{2}=T_{1}+\Delta T, T_{3}=T_{2}+$ $\Delta T, \ldots, T_{n}=T_{n-1}+\Delta T$ which are separated by the time interval $\Delta T=4 \times 10^{-2}$ (in dimensionless units) in order to decorrelate two consecutive fields. The resulting distributions approximate Poisson probability distributions, whose peak lies below $q=1$, and appears to draw closer to 1 for growing $R$. At smaller $R$, we observed different kinds of distributions, such as those of Figs. 3 and 4 for $N=24$ and $N=48$, respectively, at $R^{2}=150$. However, in all cases we checked, the distribution approximates a Poisson distribution for growing $R$. For instance, in the case of

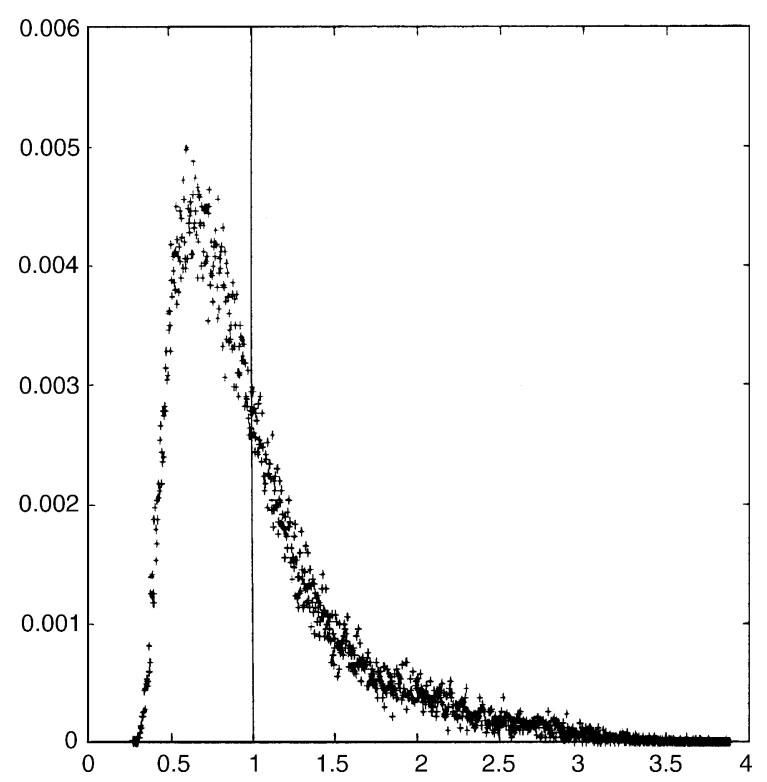

Fig. 1. Probability distribution of $\mathcal{Q}\left(\mathbf{u}^{\mathrm{NS}}\left(T_{i}\right)\right) /\langle\mathcal{Q}\rangle_{T}^{\mathrm{NS}}$ at $N=24$ and $R^{2}=200$.

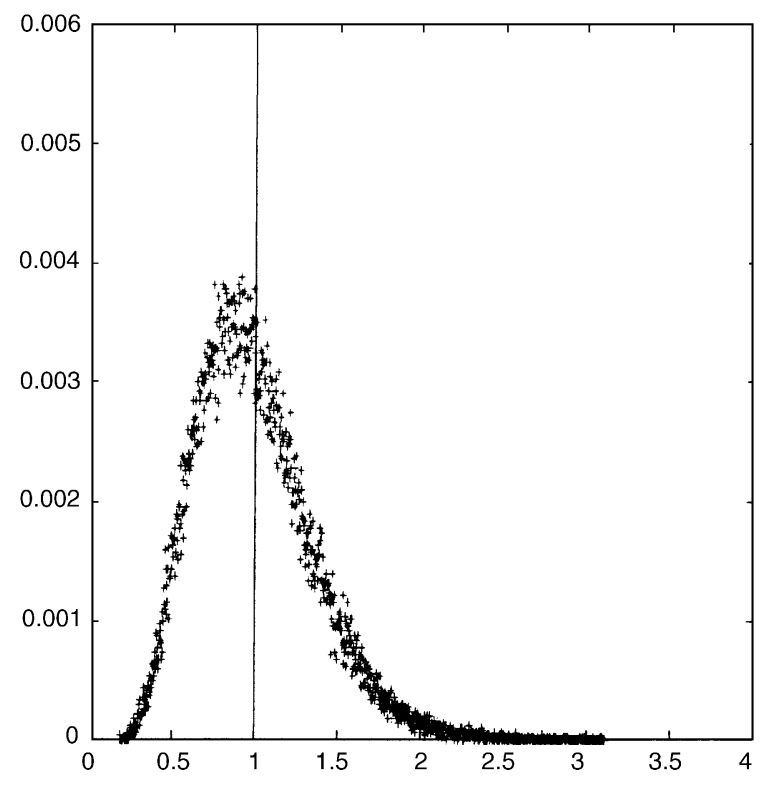

Fig. 2. Same as Fig. 1 at $R^{2}=2000$.

Fig. 4, the two peaks approach one another and finally merge when $R$ becomes sufficiently large.

Figs. 5 and 6 show the dependence of $\left\langle\alpha_{\mathcal{Q}}\right\rangle_{T}^{\mathrm{GNS}}$ on the variable $q$, for different values of the Reynolds number $R$ and of the spectral resolution $N$. Each

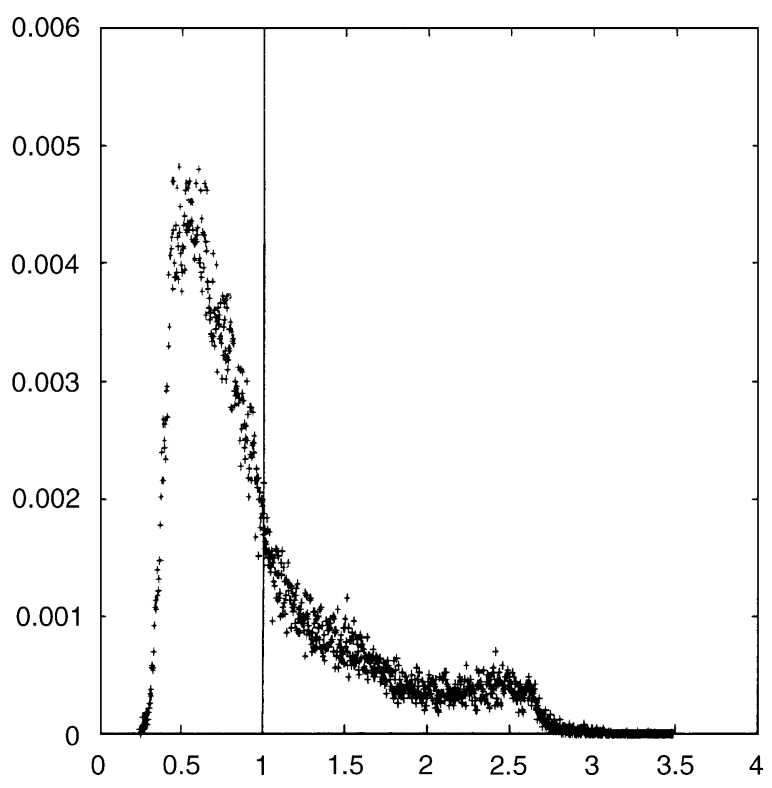

Fig. 3. Probability distribution of $\mathcal{Q}\left(\mathbf{u}^{\mathrm{NS}}\left(T_{i}\right)\right) /\langle\mathcal{Q}\rangle_{T}^{\mathrm{NS}}$ at $N=24$ and $R^{2}=150$. 


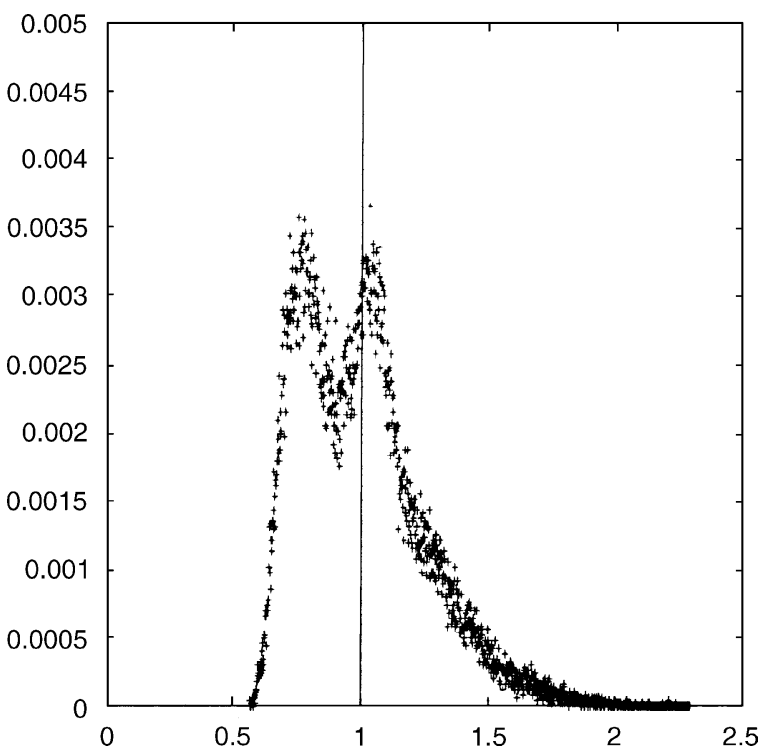

Fig. 4. Same as Fig. 3 at $N=48$.

figure contains 200 points corresponding to 200 different initial fields $\mathbf{u}^{\mathrm{NS}}\left(T_{j}\right)$. The data in Figs. 5 and 6 show a sharp, albeit not simple, connection between the average values $\left\langle\alpha_{\mathcal{Q}}\right\rangle_{T}^{\mathrm{GNS}}$ and the constant enstrophy of the corresponding GNS evolutions. Sim-

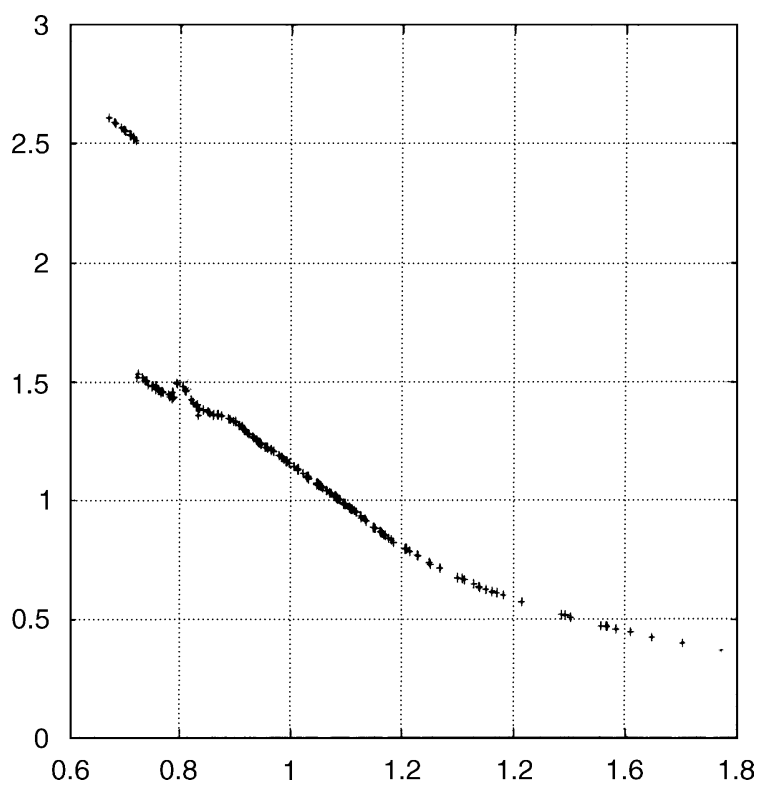

Fig. 5. Average $\left\langle\alpha_{\mathcal{Q}}\right\rangle_{T}^{\mathrm{GNS}}$ as a function of $\mathcal{Q}\left(\mathbf{u}^{\mathrm{NS}}\left(T_{i}\right)\right) /\langle\mathcal{Q}\rangle_{T}^{\mathrm{NS}}$ for $N=48$ and $R^{2}=122$.

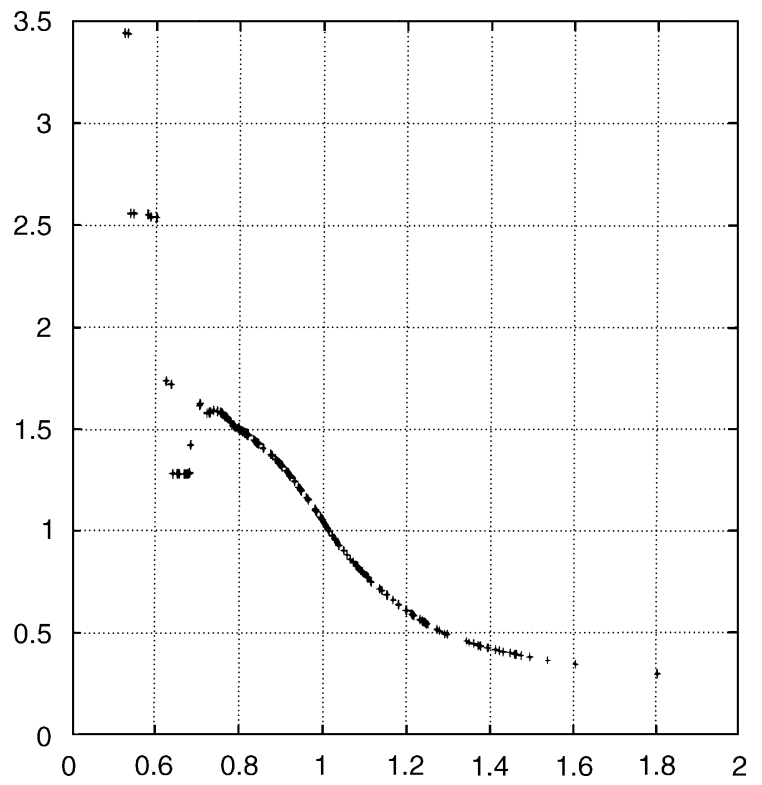

Fig. 6. Same as Fig. 5 at $N=120$ and $R^{2}=150$.

ilarly to the case of Ref. [4], this relation often yields $\left\langle\alpha_{\mathcal{Q}}\right\rangle_{T}^{\mathrm{GNS}} \approx 1$ for $q=1$, but not always, indicating that at finite $N$ and $R,\left\langle\alpha_{\mathcal{Q}}\right\rangle_{T}^{\mathrm{GNS}}$ depends on all the parameters $N, R$ and $q$ in a way which is difficult to characterize. As mentioned above, this dependence may be due to the coexistence of steady states with different statistical properties which, then, makes difficult a direct test of the Equivalence Conjecture.

To clarify this issue, one should analyze a two parameters space for the GNS evolution, considering many spectral resolutions $N$ and, for each $N$, many Reynolds numbers $R$. This is numerically too expensive; therefore, we concentrated on the case with $N=$ 24 , which can be controlled quite well numerically. We considered a set of pairs $\left(R^{2}, \mathcal{Q}_{1}\right)$, and for each of them we took a number $n_{i c}$ of initial fields sampled at random with an absolutely continuous (with respect to the Lebesgue measure) distribution on the invariant ellipsoids identified by $\mathcal{Q}(\mathbf{u})=\mathcal{Q}_{1}$. Then, in each ellipsoid, we computed the time averages of several observables along the corresponding $n_{i c}$ GNS evolutions. In particular, we took $n_{i c}=100$, and we computed the values of $\left\langle\alpha_{\mathcal{Q}}\right\rangle_{T}^{\mathrm{GNS}}$, of $\left\langle Q_{m}\right\rangle_{T}^{\mathrm{GNS}}$ for several $m$ 's, and of $\left\langle x_{\mathbf{k}}^{2}\right\rangle_{T}^{\mathrm{GNS}}$ or $\left\langle y_{\mathbf{k}}^{2}\right\rangle_{T}^{\mathrm{GNS}}$ for several wave vectors $\mathbf{k} \in \mathcal{L}^{+}$. In some cases, all the $n_{i c}$ average 
Table 1

Number of steady states for the GNS dynamics with $N=24$ and different values of $R^{2}$ and of the fixed enstrophy $\mathcal{Q}_{1}{ }^{\text {a }}$

\begin{tabular}{lllllll}
\hline$R^{2}$ & $\mathcal{Q}_{1}=0.02$ & $\mathcal{Q}_{1}=0.1$ & $\mathcal{Q}_{1}=0.2$ & $\mathcal{Q}_{1}=0.3$ & $\mathcal{Q}_{1}=0.4$ & $\mathcal{Q}_{1}=0.5$ \\
\hline 1000 & - & 1 & 1 & 1 & $\mathrm{~S}$ & $\mathrm{~S}$ \\
1500 & - & 1 & 1 & $\mathrm{~S}$ & $\mathrm{~S}$ & $\mathrm{~S}$ \\
2000 & - & 1 & $\mathrm{~S}$ & $\mathrm{~S}$ & $\mathrm{~S}$ & $\mathrm{~S}$ \\
2500 & - & 1 & $\mathrm{~S}$ & $\mathrm{~S}$ & $\mathrm{~S}$ & $\mathrm{~S}$ \\
3000 & 1 & $\mathrm{~S}$ & $\mathrm{~S}$ & - & - & - \\
3500 & 1 & $\mathrm{~S}$ & - & - & - & - \\
4000 & 1 & $\mathrm{~S}$ & - & - & - & - \\
4500 & 1 & $\mathrm{~S}$ & - & - & - & - \\
5000 & 1 & $\mathrm{~S}$ & - & - & & - \\
\hline
\end{tabular}

${ }^{a}$ The symbol 1 denotes the cases with just one steady state, while $\mathrm{S}$ denotes the cases with more than one steady state.

values of each observable turned out to be equal up to numerical errors; in other cases there was at least one observable whose $n_{i c}$ averages were not all the same. We take the first as cases in which there is a unique steady state which attracts all initial conditions in the phase space, while the other cases clearly correspond to situations in which two or more steady states coexist, and hysteresis phenomena are possible [11].

Our results are summarized in Table 1 , where 1 denotes the cases for which only one steady state was evidenced by our calculations, while S (for "several") indicates the cases with more than one steady state. Table 1 shows that for fixed $\mathcal{Q}_{1}$, small $R^{2}$ implies the presence of a unique steady state, while growing $R^{2}$ implies the coexistence of a growing number of steady states. Similarly, for fixed $R^{2}$, there is only one steady state if $\mathcal{Q}_{1}$ is small, while there is a growing number of steady states if $\mathcal{Q}_{1}$ grows. The cases denoted by a dash have not been investigated, due to the length of the relevant simulations but, extrapolating our results to such cases, it seems reasonable to conclude that we should have $S$ 's in place of the dashes for $\mathcal{Q}_{1}=$ $0.2,0.3,0.4,0.5$, and 1's for $\mathcal{Q}_{1}=0.02$.

To clarify these facts, let us consider some example more in detail.

\subsection{Case with $\mathcal{Q}_{1}=0.1$}

Table 2 shows the dependence of $\bar{\alpha}_{\mathcal{Q}}$ on $R^{2}$ for $\mathcal{Q}_{1}=0.1$, where

$\bar{\alpha}_{\mathcal{Q}}=\frac{1}{n_{i c}} \sum_{j=1}^{n_{i c}}\left\langle\alpha_{\mathcal{Q}}\right\rangle_{T}^{\mathrm{GNS}}\left(\mathbf{u}_{j}^{\mathrm{GNS}}(0)\right)$, $n_{i c}$ is the number of initial conditions $\mathbf{u}_{j}^{\mathrm{GNS}}(0)$ for which $\left\langle\alpha_{\mathcal{Q}}\right\rangle_{T}^{\mathrm{GNS}}$ is computed, and $\sigma\left(\bar{\alpha}_{\mathcal{Q}}\right)$ is the standard deviation of the distribution of the $\langle\alpha\rangle_{T}^{\mathrm{GNS}}$ values.

These results show how the relative spreading of the $\left\langle\alpha_{\mathcal{Q}}\right\rangle_{T}^{\text {GNS }}$ values has a sudden jump passing from the case of a unique steady state to the case of coexisting steady states. In the first case, the relative spreading is only an indication of the numerical error on the calculation of $\left\langle\alpha_{\mathcal{Q}}\right\rangle_{T}^{\mathrm{GNS}}$, as evidenced also by the results for $R^{2}=1500,2000$ obtained from simulations of different length, and by the fact that $\sigma\left(\bar{\alpha}_{\mathcal{Q}}\right) / \bar{\alpha}_{\mathcal{Q}}$ does not change much with $R^{2}$. Differently, in the second case, we observe that the relative spreading rapidly grows with $R^{2}$, in a monotonic fashion.

Table 2

Properties of $\bar{\alpha}_{\mathcal{Q}}$ as a function of $R^{2}$, for two values of the integration time $T$, where needed ${ }^{\mathrm{a}}$

\begin{tabular}{rllll}
\hline$R^{2}$ & $\bar{\alpha}_{\mathcal{Q}}$ & $\sigma\left(\bar{\alpha}_{\mathcal{Q}}\right)$ & $T$ & $\sigma\left(\bar{\alpha}_{\mathcal{Q}}\right) / \bar{\alpha}_{\mathcal{Q}}$ \\
\hline 300 & 0.443966 & $1.29476 \times 10^{-3}$ & 104 & $2.92 \times 10^{-3}$ \\
350 & 0.393078 & $1.03238 \times 10^{-3}$ & 104 & $2.63 \times 10^{-3}$ \\
400 & 0.352696 & $9.13097 \times 10^{-4}$ & 104 & $2.59 \times 10^{-3}$ \\
450 & 0.319712 & $1.11033 \times 10^{-3}$ & 104 & $3.47 \times 10^{-3}$ \\
500 & 0.291373 & $9.46472 \times 10^{-4}$ & 104 & $3.25 \times 10^{-3}$ \\
550 & 0.267641 & $9.64748 \times 10^{-4}$ & 104 & $3.6 \times 10^{-3}$ \\
1000 & 0.153389 & $5.29941 \times 10^{-4}$ & 104 & $3.45 \times 10^{-3}$ \\
1500 & 0.103519 & $4.72735 \times 10^{-4}$ & 104 & $4.57 \times 10^{-3}$ \\
1500 & 0.103682 & $2.65998 \times 10^{-4}$ & $3 \times 10^{4}$ & $2.56 \times 10^{-3}$ \\
2000 & $7.823 \times 10^{-2}$ & $3.3869 \times 10^{-4}$ & 104 & $4.33 \times 10^{-3}$ \\
2000 & $7.8126 \times 10^{-2}$ & $2.531 \times 10^{-4}$ & $3 \times 10^{4}$ & $3.24 \times 10^{-3}$ \\
2500 & $6.2523 \times 10^{-2}$ & $1.98 \times 10^{-4}$ & $3 \times 10^{4}$ & $3.17 \times 10^{-3}$ \\
3000 & $4.783 \times 10^{-2}$ & $2.9457 \times 10^{-3}$ & 104 & $6.16 \times 10^{-2}$ \\
4000 & $2.129 \times 10^{-2}$ & $1.476 \times 10^{-2}$ & 104 & $6.92 \times 10^{-1}$ \\
5000 & 0.160473 & $7.080 \times 10^{-3}$ & 104 & $4.4 \times 10^{-2}$ \\
6000 & $4.11 \times 10^{-3}$ & $6.1375 \times 10^{-3}$ & 104 & 1.49 \\
\hline
\end{tabular}

${ }^{\mathrm{a}}$ Here, $n_{i c}=20$ for $R^{2} \in[300,550], n_{i c}=100$ for $R^{2} \geq 1000$. 


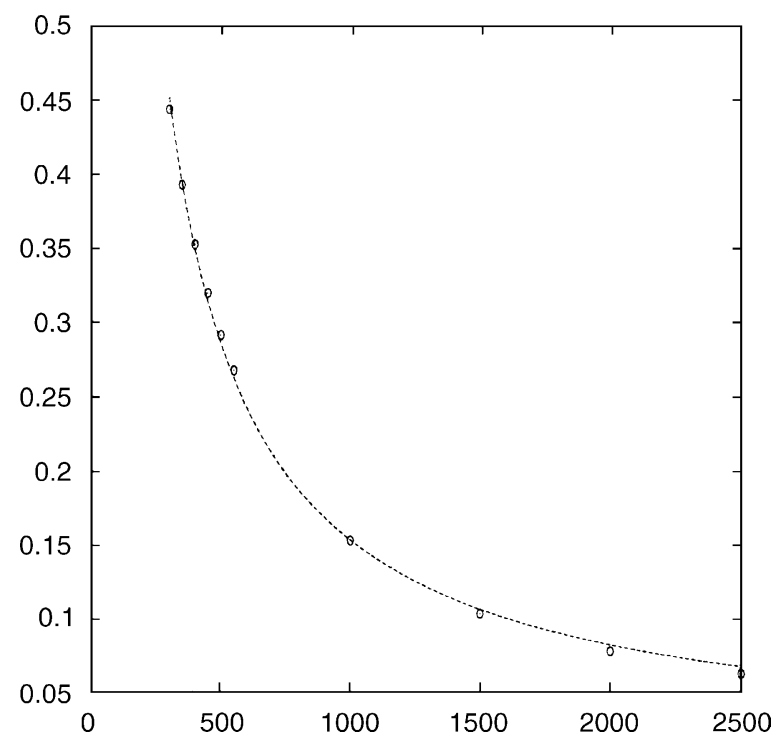

Fig. 7. Average $\left\langle\alpha_{\mathcal{Q}}\right\rangle_{T}^{\mathrm{GNS}}$ as a function of $R^{2}$, together with the fit $f(x)=75.5829 / R^{b}, b=1.7946$, for $N=24$ and $\mathcal{Q}_{1}=0.1$.

Fig. 7 reports a fit of the values of $\bar{\alpha}_{\mathcal{Q}}$ as a function of $R^{2}$ in the range with a unique steady state. Because $\alpha_{\mathcal{Q}}$ is a kind of viscosity, if the conjectured equivalence between the NS and the GNS dynamics really holds, as the numerical results of [4,5] suggest, one should find that $\left\langle\alpha_{\mathcal{Q}}\right\rangle^{\mathrm{GNS}} \sim 1 / R$. The spectral resolution we use is clearly insufficient to correctly represent this kind of hydrodynamic properties for the values of $R$ we considered. In fact, the best fit of our data yields $\left\langle\alpha_{\mathcal{Q}}\right\rangle_{T}^{\mathrm{GNS}}=$ const. $/ R^{b}$ with $b \approx 1.7946$.

Figs. $8-10$ show the time evolution of $\alpha_{\mathcal{Q}}$. As $R$ grows, the evolution changes from apparently chaotic with a unique steady state, to quasi-periodic or intermittent, depending on the initial condition. The chaotic steady state for $R^{2}=1000$, has a Lyapunov spectrum with 10 positive exponents (the maximum being $\lambda_{\max } \approx 33.81$ ) and Kaplan-Yorke dimension $D_{\mathrm{KY}} \approx$ $22.588[12]$.

For the case with $R^{2}=3000$, out of the 100 different initial conditions which we considered, 98 yield intermittent evolutions with $\left\langle\alpha_{\mathcal{Q}}\right\rangle_{T}^{\mathrm{GNS}} \in[0.040,0.048]$, one initial condition yields a quasi-periodic evolution with $\left\langle\alpha_{\mathcal{Q}}\right\rangle_{T}^{\mathrm{GNS}} \approx 0.0048$, and the last initial condition leads to a long erratic transient, apparently followed

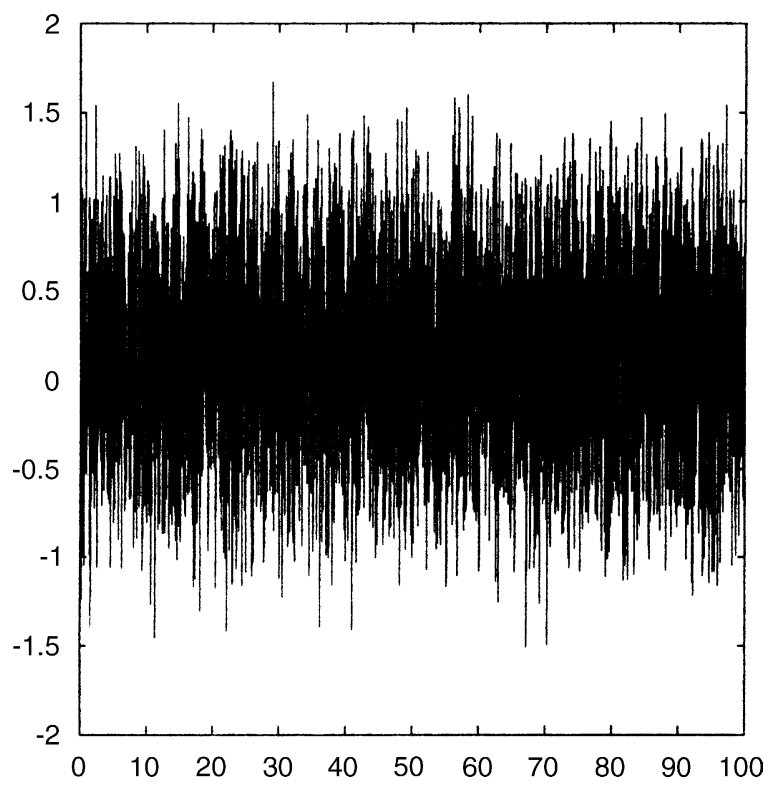

Fig. 8. Quantity $\alpha_{\mathcal{Q}}$ as a function of time for the chaotic case at $N=24, R^{2}=1000$ and $\mathcal{Q}_{1}=0.1$.

by an intermittent steady state with $\left\langle\alpha_{\mathcal{Q}}\right\rangle_{T}^{\mathrm{GNS}} \approx 0.051$. However, the length of the transient casts some doubts on the sufficiency of our simulation times to correctly understand this case. The intermittent cases, includ-

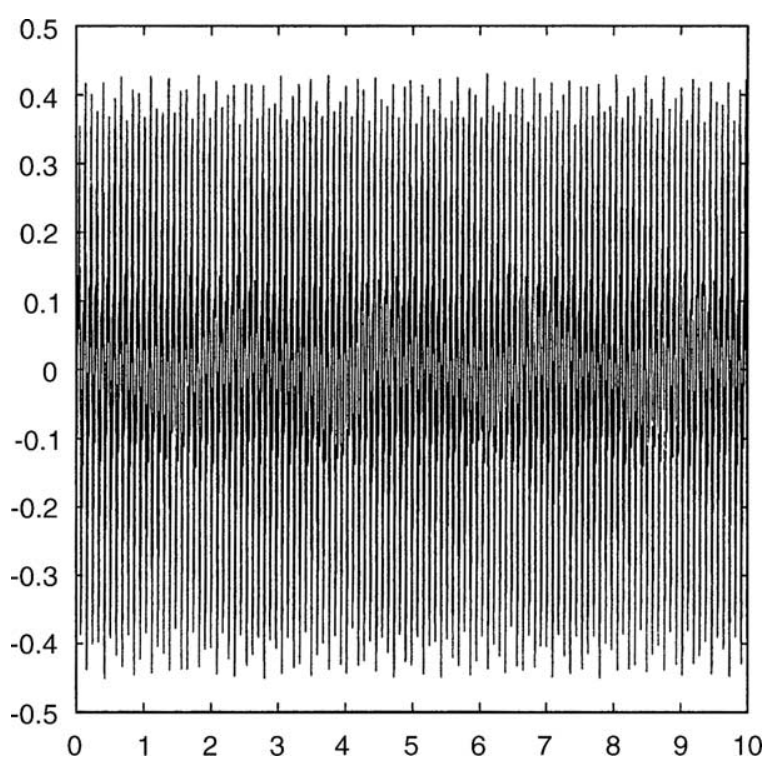

Fig. 9. Same as Fig. 8 for the quasi-periodic case at $N=24$, $R^{2}=3000$ and $\mathcal{Q}_{1}=0.1$. 


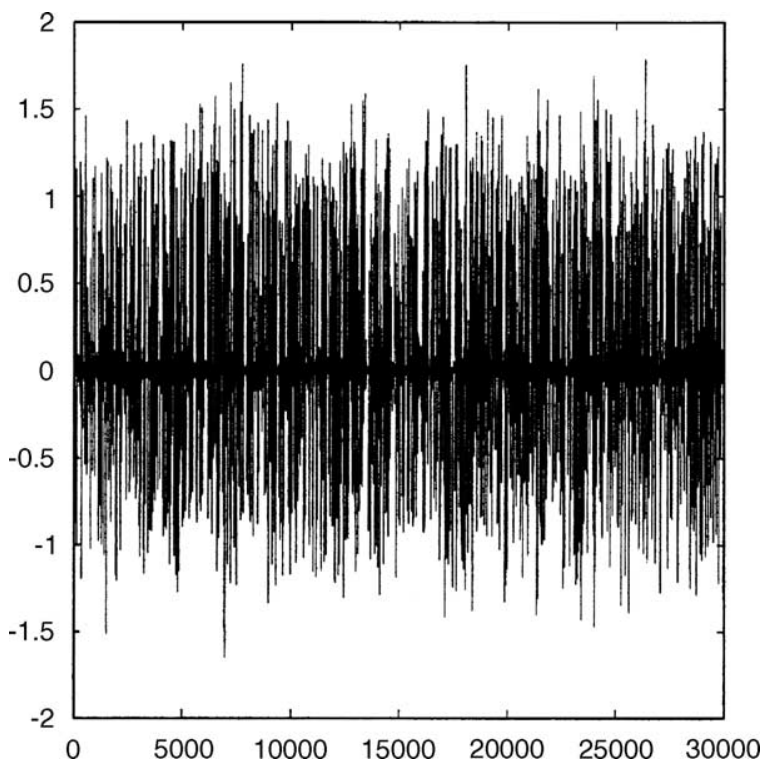

Fig. 10. Same as Fig. 8 for the intermittent case at $N=24$, $R^{2}=3000$ and $\mathcal{Q}_{1}=0.1$.

ing this last dubious case, are also characterized by fluctuations of $\alpha_{\mathcal{Q}}$ with amplitude which varies over two orders of magnitude.

It is interesting to observe that a further increase of $R$ can result in a suppression of the intermittent behavior. For instance, at $R^{2}=4500$ we found that three quasi-periodic steady states coexist, and that every initial condition we considered was attracted by one of them, suggesting that the phase space does not contain any other steady state. This implies that larger $R$ does not produce a higher degree of chaos in the GNS dynamics, if the spectral resolution $N$ is fixed. On the contrary, larger $R$ at fixed $N$ leads to a higher degree of order.

The situation appears different when the spectral resolution $N$ is not fixed but grows with $R$. For instance, the case with $\mathcal{Q}_{1}=0.1, R^{2}=3000$ and $N=48$ yields a unique steady state, with $\left\langle\alpha_{\mathcal{Q}}\right\rangle_{T}^{\mathrm{GNS}} \in$ $[0.05195,0.05402]$ where the width of the interval basically indicates the numerical error on the computed value of $\left\langle\alpha_{\mathcal{Q}}\right\rangle_{T}^{\mathrm{GNS}}$. Similarly, for $N=80$ and same $R^{2}$ and $\mathcal{Q}_{1}$, we obtained a unique steady state with $\left\langle\alpha_{\mathcal{Q}}\right\rangle_{T}^{\mathrm{GNS}} \in[0.02030,0.02095]$. The same holds for the other averages which we computed to test the

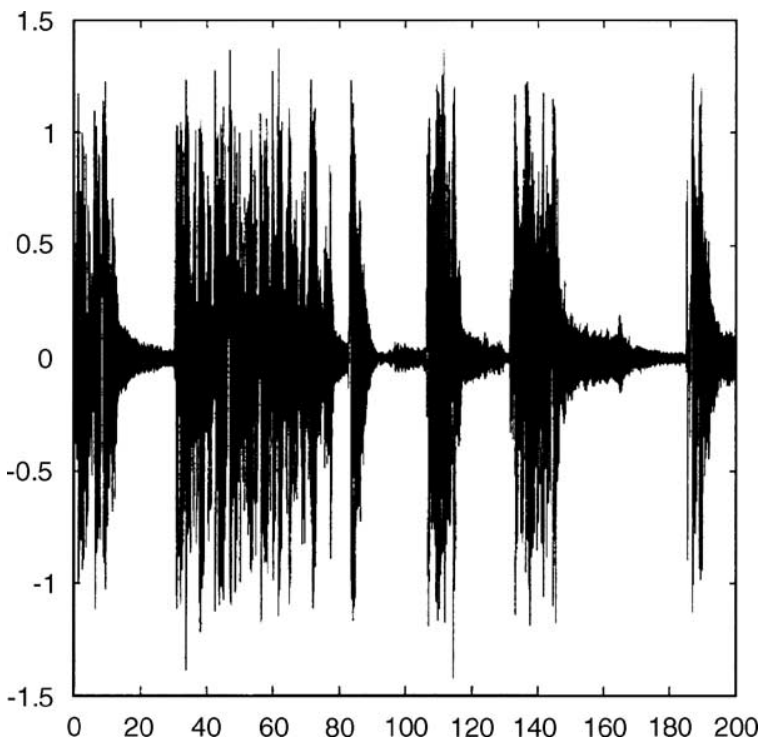

Fig. 11. Same as Fig. 8 for the intermittent case at $N=24$, $R^{2}=2000$ and $\mathcal{Q}_{1}=0.2$.

uniqueness of the steady states, some of which, like $\alpha_{\mathcal{Q}}$, turn out to be approximately Gaussian, while the others, like the real or imaginary parts of some Fourier modes of the velocity field, are not Gaussian. In particular, the non-Gaussian distributions include bimodal distributions which are symmetric about 0 .

\subsection{Cases with $\mathcal{Q}_{1}=0.2$ and $\mathcal{Q}_{1}=0.4$}

The cases with $N=24, \mathcal{Q}_{1}=0.2$ and $R^{2}=$ 2000, 3000 also have intermittent and quasi-periodic steady states, which are selected by the initial conditions. This is exemplified by Figs. 11 and 12. Fig. 13 is the projection of the coordinates $x_{(0,1)}$ and $x_{(1,2)}$ when $x_{(1,-1)}=0$ for $R^{2}=2000$, and Fig. 14 is the projection of the coordinates $x_{(0,1)}$ and $x_{(2,0)}$ when $x_{(1,-1)}=0$ for $R^{2}=3000$. In both cases we have a quasi-periodic evolution and, in particular, the case with $R^{2}=3000$ has at least three different quasi-periodic steady states. The power spectrum of the signal of Fig. 12, reported in Fig. 15, contains at least two different basic frequencies and some of their integer combinations. The computation of 


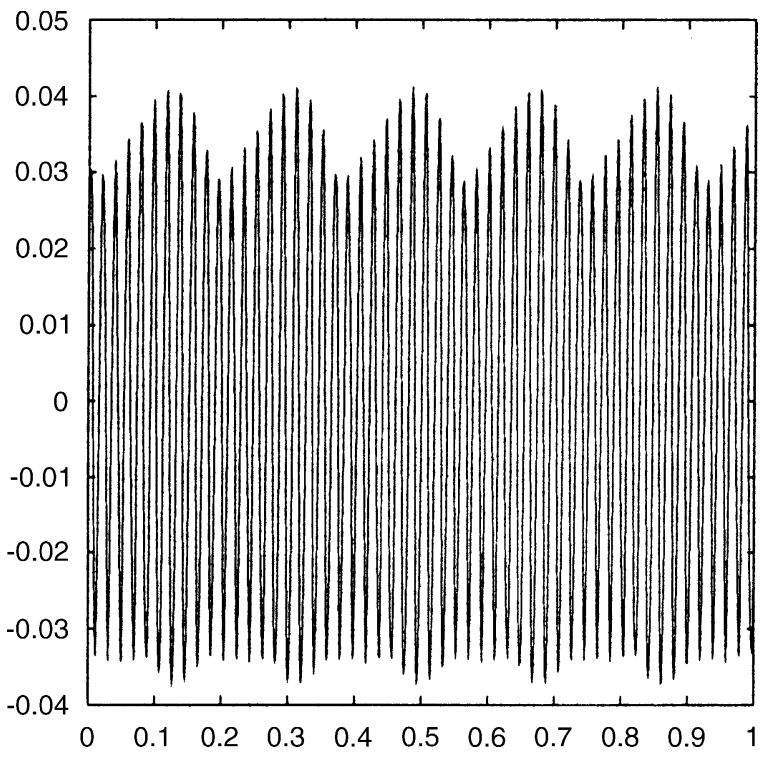

Fig. 12. Same as Fig. 8 for the quasi-periodic case at $N=24$, $R^{2}=2000$ and $\mathcal{Q}_{1}=0.2$.

the Lyapunov spectrum of this steady state is quite delicate and, in order to obtain what we consider sufficiently accurate data (see point 2 in Section 4), a time step of $10^{-4}$ was used. The resulting spectrum has four negative exponents of magnitude of order

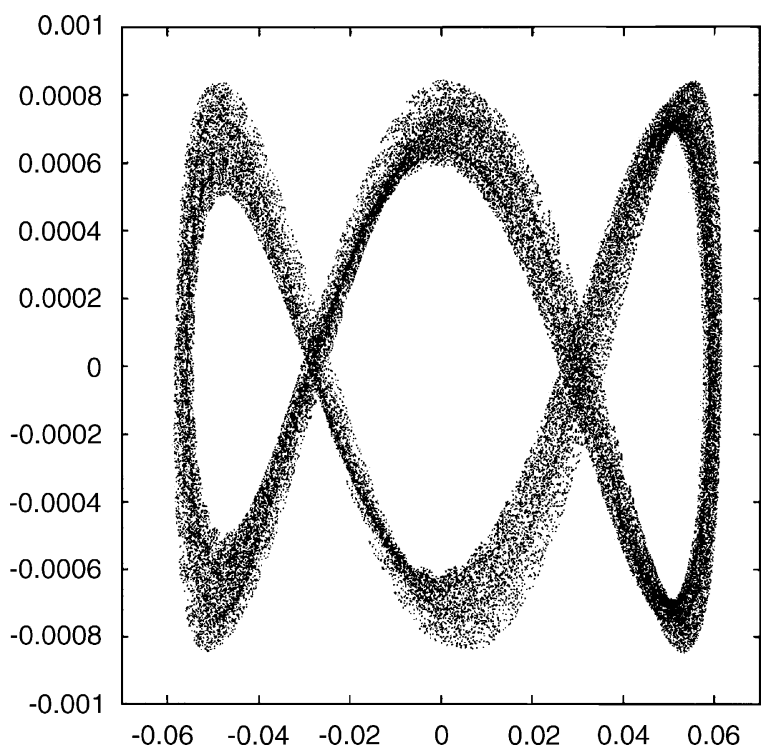

Fig. 13. Coordinates $x_{(0,1)}$ and $x_{(1,2)}$ for $x_{(1,-1)}=0$ for the quasi-periodic case at $N=24, R^{2}=2000$ and $\mathcal{Q}_{1}=0.2$.

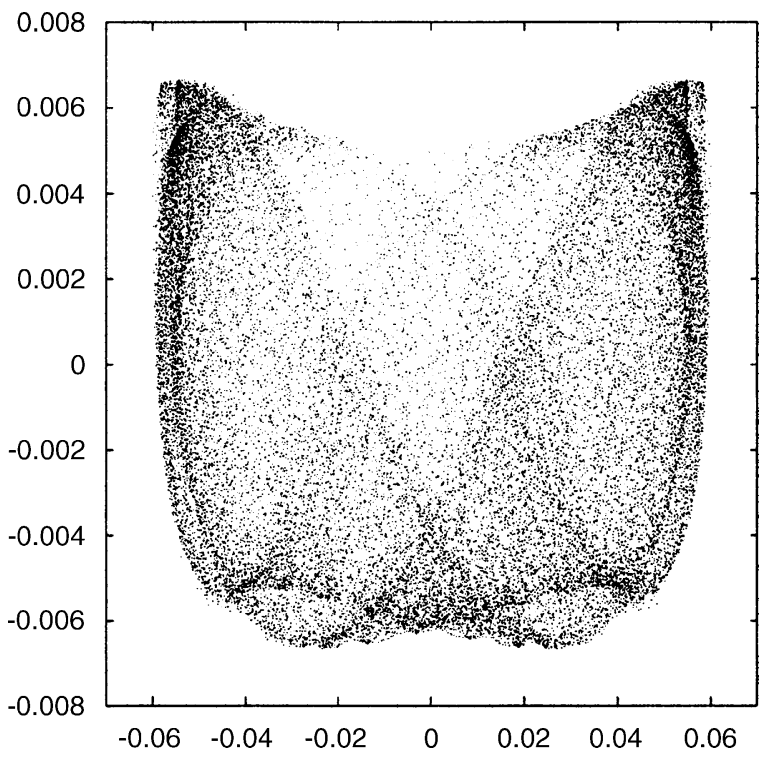

Fig. 14. Coordinates $x_{(0,1)}$ and $x_{(2,0)}$ for $x_{(1,-1)}=0$ for the quasi-periodic case at $N=24, R^{2}=3000$ and $\mathcal{Q}_{1}=0.2$.

$\mathrm{O}\left(10^{-3}\right)$, while the other exponents have magnitude ranging from $\mathrm{O}\left(10^{-4}\right)$ down to $\mathrm{O}\left(10^{-6}\right)$, and cannot be distinguished from zero with our accuracy. The cases with $N=24, \mathcal{Q}_{1}=0.4$ and $R^{2}=3000$ have quasi-periodic, intermittent and chaotic steady

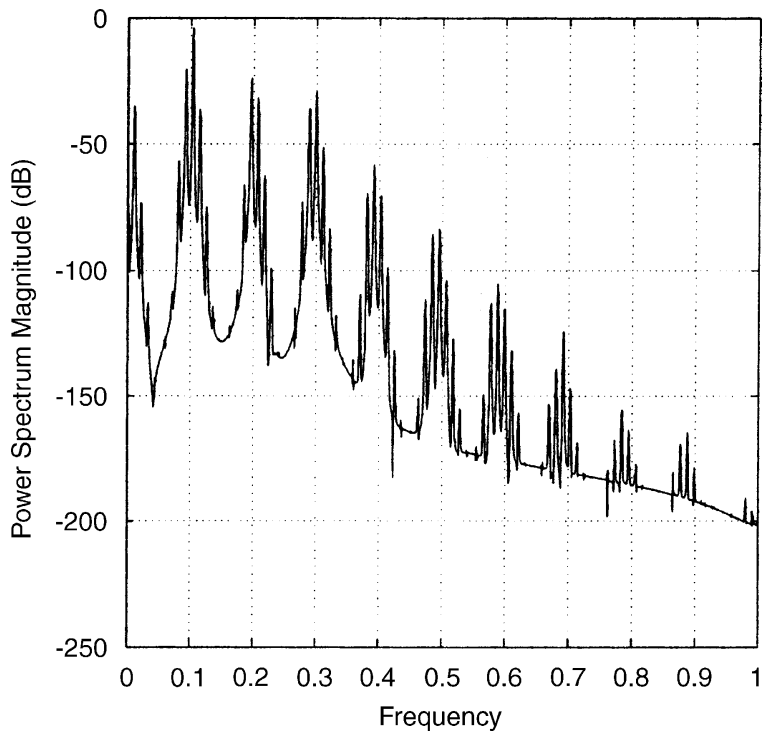

Fig. 15. Power spectrum of $\alpha_{\mathcal{Q}}$ for the case of Fig. 12. 


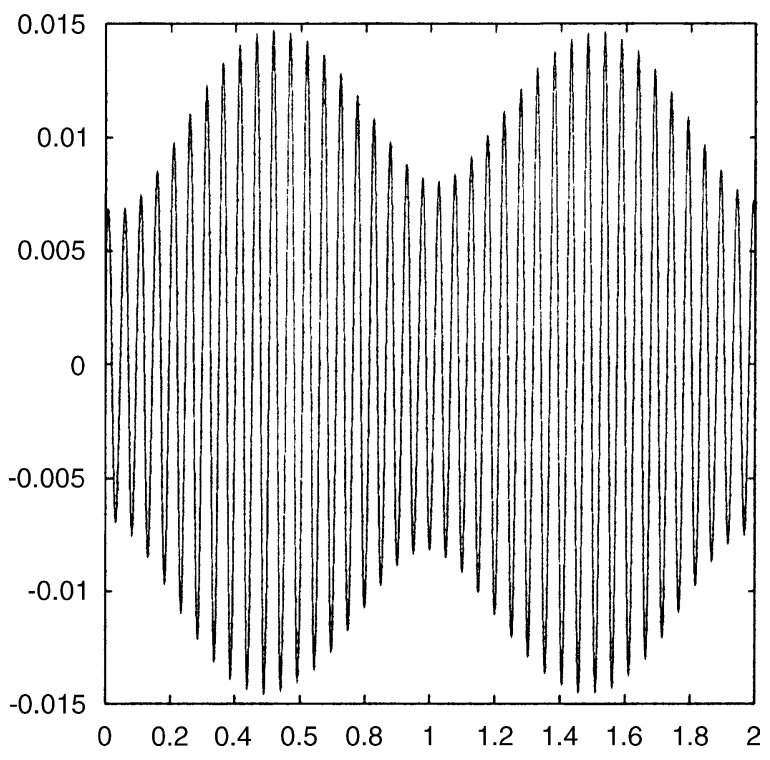

Fig. 16. $\alpha_{\mathcal{Q}}$ as a function of time for the quasi-periodic case at $N=24, R^{2}=3000$ and $\mathcal{Q}_{1}=0.4$.

states, selected by the initial condition, as evidenced by the time dependence of $\alpha_{\mathcal{Q}}$ shown in Figs. 16-18. This distinguishes this case from the previous ones, which were chaotic only when the steady state was unique.

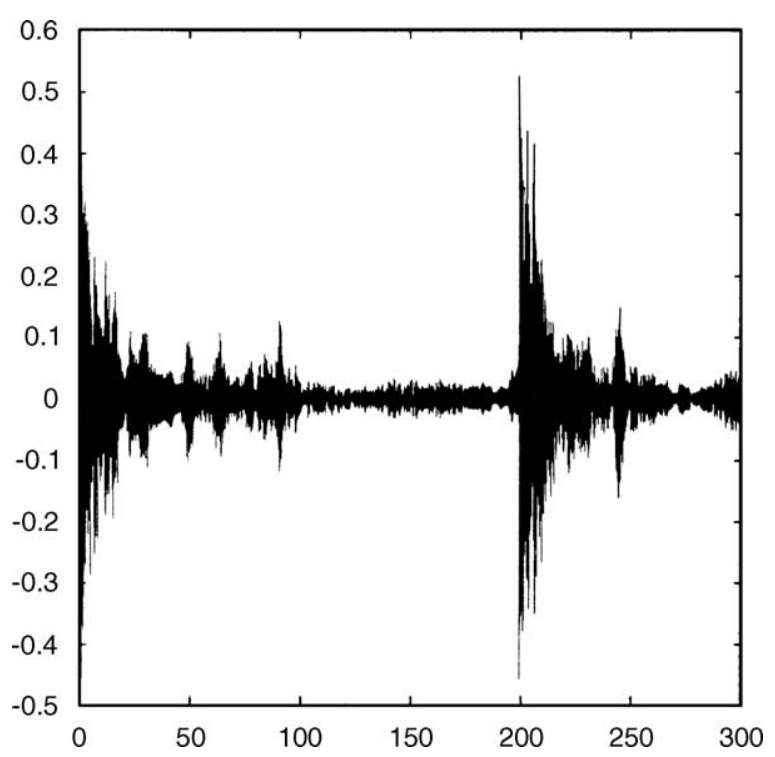

Fig. 17. $\alpha_{\mathcal{Q}}$ as a function of time for the intermittent case at $N=24, R^{2}=3000$ and $\mathcal{Q}_{1}=0.4$.

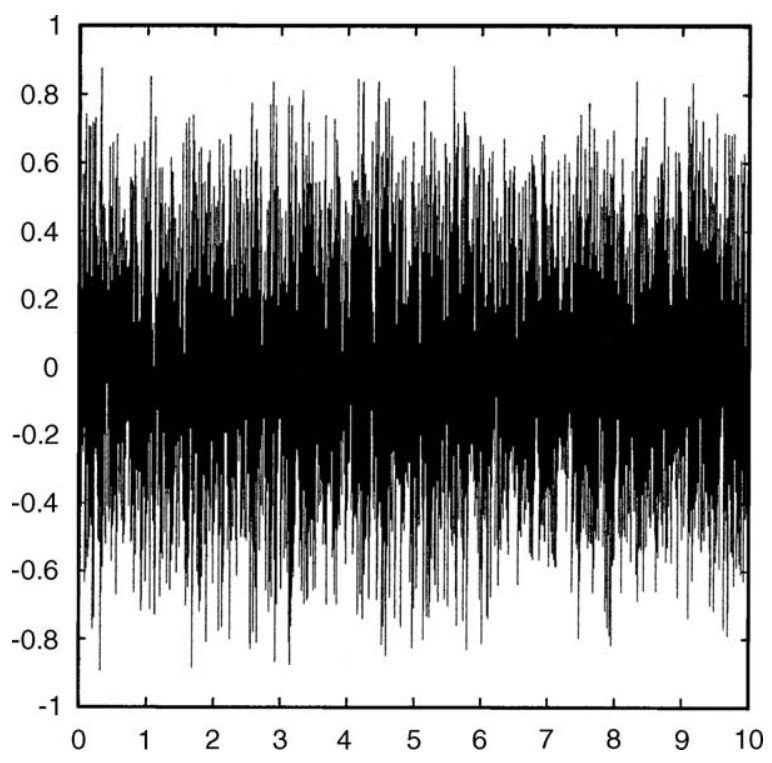

Fig. 18. $\alpha_{\mathcal{Q}}$ as a function of time for the chaotic case at $N=24$, $R^{2}=3000$ and $\mathcal{Q}_{1}=0.4$.

\section{Concluding remarks}

1. Our study of the GNS dynamical system has evidenced that fixing the spectral resolution $N$ and the enstrophy $\mathcal{Q}$, and letting $R$ grow, the dynamics gradually become more and more ordered. This is a consequence of the fact that the Gaussian constraint has to work more for larger $R$ to keep $\mathcal{Q}$ fixed at the same value $\mathcal{Q}_{1}$. The corresponding evolutions, then dissipate more and the contraction of the phase space volumes is stronger. Therefore, the steady states occupy a small fraction of the phase space and make room for different steady states to coexist.

If $\mathcal{Q}_{1}$ decreases at fixed $N, R$, the opposite happens: the steady states become less ordered, and occupy larger fractions of the phase space, leaving no room for the coexistence of different steady states. This is due to the fact that smaller $\mathcal{Q}_{1}$ implies that the Gaussian constraint works less.

Counterintuitive looks the fact that increasing the spectral resolution $N$ with fixed $R$ and $\mathcal{Q}$, i.e. increasing the volume of the phase space, leads to a unique steady state, rather than to an increase in the number of coexisting steady states. This is 
due to the fact that the denominator of (7) grows more rapidly with $N$ than the numerator does, because the denominator contains higher powers of $\mathbf{k}$ than the numerator. The result is that the dissipation per degree of freedom needed to constrain $\mathcal{Q}$ decreases as $N$ grows, then the phase space contraction rate decreases leaving no room for more than one steady state. This suggests that there must be a scaling $N=N\left(R ; \mathcal{Q}_{1}\right)$ for the GNS equations which determines the boundary between the cases whose steady states are unique and the cases whose phase space is decomposed in various ergodic components.

The main consequence of the existence of this scaling is that the Equivalence Conjecture appears to be valid for the NS and GNS dynamics, in agreement with the results of Refs. [4,5], but some care must be used in order to observe it. In practice, the equivalence between NS and GNS dynamics can be observed for growing $R$ only if the simulations are performed with finer and finer spectral resolution $\hat{N}>N\left(R ; \mathcal{Q}_{1}\right)$. The need for $N$ to be "much larger than the Kolmogorov scale", so that the cut off equations be physically relevant, was already discussed in [2], but the precise form of $N\left(R ; \mathcal{Q}_{1}\right)$ is still unknown. Indeed, the present work constitutes the first quantitative study of the existence of $N\left(R ; \mathcal{Q}_{1}\right)$ for the GNS dynamics. The phenomenon by which higher $R$ at fixed $N, \mathcal{Q}_{1}$, or higher $\mathcal{Q}_{1}$ at fixed $N, R$, lead to more orderly dynamics looks similar to the phenomenon which produces "string phases" in non-equilibrium molecular dynamics [13].

Alternatively, the validity of the Equivalence Conjecture can be assessed only restricting the analysis to the basins of attraction of each separate steady state. As a matter of fact, this is the procedure developed in [4], and used also in [5], in order to deal with the cases in which the steady states are unique as well as with the cases characterized by several coexisting steady states.

2. The purpose of our calculations of the Lyapunov exponents of the GNS dynamics was to distinguish the chaotic cases from the quasi-periodic ones. Therefore, we were not concerned with the plainly positive or negative exponents, as much as we were with the vanishing ones. It is for this reason that we used the presence of at least two "vanishing" exponents as the criterion to assess the accuracy of our calculations. In other words. the accuracy of the calculation of the Lyapunov exponents was kept under control making sure that at least two zero exponents $\lambda_{k}, \lambda_{k+1}$, corresponding to the direction of the flow and to the conservation of $\mathcal{Q}$, were present in the spectrum. The test consisted in checking that $\left|\lambda_{k}\right|$ and $\left|\lambda_{k+1}\right|$ be less than a given tolerance $\delta \lambda$, which we choose to be of order $\mathrm{O}\left(10^{-4}\right)$.

Nevertheless, such calculations remain particularly delicate in the cases which we qualified as quasi-periodic, due to the presence of a number of very small Lyapunov exponents, whose actual values cannot be distinguished from zero with our accuracy.

This is a common problem when dealing numerically with zero exponents [5], if the classical algorithm developed in Ref. [14] is used to calculate the whole Lyapunov spectrum, as appropriate in our case. Therefore, in the quasi-periodic cases, some uncertainty remains on whether some of the exponents are really zero or small but positive (the negative exponents do not matter in this instance). This prevents us from stating with absolute certainty that the cases we took as quasi-periodic are really such. However, all the information we have, including the power spectra and the properties of the projected phase space portraits, support the view that those steady states are quasi-periodic, and that their largest exponents are not positive. Even if this was not the case, the time scales over which the chaotic behavior could become evident are so long to make such a behavior not distinguishable from numerical noise, hence irrelevant for any practical observation.

One interesting observation is that the number of "vanishing" Lyapunov exponents, and the projected phase space portraits, suggest that our quasiperiodic steady states have at least three independent frequencies, in contrast with the cases studied by Franceschini, Zanasi and one of us in $[15,16]$, whose independent frequencies are not more than 3 . 
Table 3

Steady states for the GNS dynamics with $N=24$ and different values of $R^{2}$ and of the fixed enstrophy $\mathcal{Q}_{1}{ }^{\text {a }}$

\begin{tabular}{llll}
\hline$R^{2}$ & $\mathcal{Q}_{1}=0.1$ & $\mathcal{Q}_{1}=0.2$ & $\mathcal{Q}_{1}=0.3$ \\
\hline 1000 & 1 & 1 & 1 \\
1500 & 1 & 1 & $(i, q)$ \\
2000 & 1 & $(i, q)$ & $(q, i)$ \\
2500 & 1 & $(q, i)$ & $q$ \\
3000 & $(i, q)$ & $q$ & $(i, q, c)$ \\
\hline
\end{tabular}

a The symbol 1 denotes the cases with one chaotic steady state, $c$ denotes the presence of chaotic steady states, $i$ the presence of intermittent steady states, and $q$ the presence of quasi-periodic steady states. The order in which the symbols $c, i$ and $q$ appear in a given box indicates the size of the relevant basins of attraction, in decreasing order (the pair $(i, q)$ says that there are more initial conditions which lead to intermittent states than initial conditions which lead to quasi-periodic states).

3. The GNS evolutions which we considered intermittent depend on the parameters $R$ and $\mathcal{Q}_{1}$ in a fashion similar to that described by the PomeauManneville scenario of intermittency [17,18], but there is at least one difference. The "regular" phases of our intermittent GNS evolutions appear to be the shadow of a quasi-periodic, not a periodic, trajectory which may exist at close parameters values. This finds support also on the results reported in Table 3, for the case with $N=24$, which suggest that the intermittency does not exist in the absence of quasi-periodic motions.

A detailed study of these aspects, and an investigation in the spirit of [19] of the possible sequence of bifurcations leading from ordered motions to chaotic ones in the GNS dynamics, is quite expensive from a numerical point of view, and will be the object of future works.

\section{Acknowledgements}

LR gratefully acknowledges support from GNFMINDAM. CG and CV were supported by the research project Cofin 2000 "Sistemi dinamici classici, quantistici e stocastici". The authors are indebted to G. Gallavotti for inspiring this work and for enlightening discussions. Thanks are in order to V. Franceschini for his help with the interpretation of some numerical results.

\section{References}

[1] G. Gallavotti, Equivalence of dynamical ensembles and Navier-Stokes equations, Phys. Lett. A. 223 (1996) 91.

[2] G. Gallavotti, Dynamical ensemble equivalence in fluid mechanics, Physica D 105 (1997) 163.

[3] C. Lanczos, The Variational Principles of Mechanics, Dover, New York, 1970.

[4] L. Rondoni, E. Segre, Fluctuations in two-dimensional reversibly damped turbulence, Nonlinearity 12 (6) (1999) 1471.

[5] G. Gallavotti, L. Rondoni, E. Segre, Lyapunov spectra and nonequilibrium ensembles equivalence in $2 \mathrm{D}$ fluid mechanics, Physica D 187 (2004) 338-357.

[6] E. Hairer, C. Lubich, G. Wanner, Geometric Numerical Integration, Springer, Berlin, 2002.

[7] G. Gallavotti, E.G.D. Cohen, Dynamical ensembles in stationary states, J. Stat. Phys. 80 (5/6) (1995) 931.

[8] G. Gallavotti, A local fluctuation theorem, J. Phys. A 263 (1999) 39.

[9] S. Ciliberto, C. Laroche, An experimental test of the Gallavotti-Cohen fluctuation theorem, J. Phys. IV 8 (6) (1998) 215.

[10] W.I. Goldburg, Y.Y. Goldschmidt, H. Kellay, Fluctuation and dissipation in liquid crystal electroconvection, Preprint, 2001.

[11] D. Ruelle, Elements of Differentiable Dynamics and Bifurcation Theory, Academic Press, Boston, 1989.

[12] J.-P. Eckmann, D. Ruelle, Ergodic theory of chaos and strange attractors, Rew. Mod. Phys. 57 (1985) 617.

[13] D.J. Evans, G.P. Morriss, Statistical Mechanics of Nonequilibrium Liquids, Academic Press, London, 1990.

[14] G. Benettin, L. Galgani, A. Giorgilli, J.M. Strelcyn, Lyapunov characteristic exponents for smooth dynamical systems and for Hamiltonian systems; a method for computing all of them. Part 1. Theory, Meccanica 15 (1980) 9;

G. Benettin, L. Galgani, A. Giorgilli, J.M. Strelcyn, Lyapunov characteristic exponents for smooth dynamical systems and for Hamiltonian systems; a method for computing all of them. Part 2. Applications, Meccanica 15 (1980) 21.

[15] V. Franceschini, R. Zanasi, Three-dimensional Navier-Stokes equations truncated on a torus, Nonlinearity 4 (1992) 189.

[16] C. Giberti, R. Zanasi, Behavior of a three-torus in truncated Navier-Stokes equations, Physica D 65 (1993) 300.

[17] Y. Pomeau, P. Manneville, Intermittent transition to turbulence in dissipative dynamical systems, Commun. Math. Phys. 74 (1980) 189.

[18] G. Gallavotti, Foundations of Fluid Mechanics, Springer, Berlin, 2002.

[19] V. Franceschini, C. Tebaldi, F. Zironi, Fixed point behavior of $N$-mode truncated Navier-Stakes equations as $N$ increases, J. Stat. Phys. 35 (1984) 387;

V. Franceschini, C. Giberti, M. Nicolini, Common periodic behavior in larger and larger truncations of the Navier-Stokes equations, J. Stat. Phys. 50 (1988) 879;

V. Franceschini, C. Giberti, Qualitative and quantitative stabilized behavior of truncated two-dimensional NavierStokes equations, Theor. Comput. Fluid Dynam. 2 (1991) 185 . 